\title{
Enseñanza de la colocación basada en la analogía del inglés
}

RYO TSUTAHARA

Universidad de Estudios Extranjeros de Tokio r.tsutahara@tufs.ac.jp

Resumen: En este trabajo, empezamos por defender la importancia del conocimiento de colocaciones para los estudiantes de ELE. La falta del conocimiento puede provocar frases que pueden ser gramaticalmente correctas, pero poco naturales como lluvia pesada. Aprendiendo colocaciones se amplía el vocabulario y se mejora la capacidad de expresión. Sin embargo, en cada lengua existen numerosas palabras que forman una gran cantidad de colocaciones, por lo que es provechoso buscar una estrategia que facilite el aprendizaje de estas. Considerando que la analogía que existe entre el español y el inglés ayuda a mejorar la enseñanza de la colocación, hemos establecido un material docente basado en esta analogía. El segundo objetivo de este trabajo es presentar este material y sus particularidades. También mostraremos los datos que justifican la eficacia de nuestra estrategia, datos que indican que la mayoría de las colocaciones en español y sus correspondientes en inglés son léxicamente paralelas.

Palabras claves: colocación, inglés, comparación, material docente, corpus

Abstract: In this paper, at first, I will try to show the importance of the knowledge of collocations for ELE students. Insufficient knowledge of collocations may cause phrases that can be grammatically correct but sound strange like lluvia pesada. By learning collocations, the students can expand their vocabulary and improve their capacity of expression. Therefore, it is useful to develop a strategy to facilitate the learning of collocations. I think that the parallels between Spanish and English could improve the teaching of collocations and I have established teaching materials based on this analogy. The second objective of this paper is to present it and show the distinctive feature of these materials. I will also show data that support the validity of the strategy, the data that indicate that the majority of the collocations in Spanish and the corresponding ones in English are lexically parallel.

Keywords: Collocation, English, comparison, teaching materials, corpus 


\section{Introducción}

El objetivo de este trabajo es presentar nuestro material docente para la enseñanza de colocaciones defendiendo la importancia de las mismas para los estudiantes de ELE. La novedad, o la particularidad principal de nuestro material será que está basado en la analogía con el inglés. Es decir, hemos intentado facilitar el aprendizaje y enseñanza de colocaciones del español aprovechando las similitudes que se encuentran entre el español y el inglés. Esta estrategia será eficaz no solo para los nativos de inglés sino también para los estudiantes no nativos de inglés porque por ejemplo en Japón, la mayoría de los estudiantes estudian inglés antes de estudiar español. Por eso pueden aprovechar la analogía del inglés, aunque no son nativos de inglés. Esta es la razón por la que ponemos ejemplos en inglés en nuestro material. Además, esta perspectiva comparativa no solo sirve para facilitar el aprendizaje sino también para prevenir colocaciones erróneas. En este trabajo, en la sección 3, también presentaremos los datos con los que argumentamos la eficacia de nuestra estrategia.

\subsection{Colocación}

En esta sección detallaremos el concepto de colocación o collocation en inglés. La colocación suele entenderse como una coocurrencia habitual de más de dos palabras. Stubbs (2001) lo define así:

Collocation. This is a lexical relation between two or more words which have a tendency to cooccur within a few words of each other in running text. For example, PROVIDE frequently occurs with words which refer to valuable things which people need, such as help and assistance, money, food and shelter, and information. These are some of the frequent collocates of the verb.

Stubbs (2001: 24)

En la tradición de la lingüística española, se da este mismo tipo de explicaciones. Por ejemplo, Írsula Peña (1992), en uno de los estudios más antiguos de colocaciones en español, define el concepto como «las combinaciones frecuentes y preferentes de dos o más palabras, que se unen en el seno de una frase para expresar determinados acontecimientos en situaciones comunicativas establecidas» (Írsula Peña, 1992: 717). En este estudio, basándonos en estos autores, trataremos las colocaciones como coocurrencias habituales o preferentes de palabras ${ }^{1}$.

\footnotetext{
${ }^{1}$ Para la discusión más detallada de la colocación, véase Sinclair (1991), Corpas Pastor (1997) y Koike (2000).
} 


\subsection{Importancia de las colocaciones}

Consideramos que el conocimiento de colocaciones es indispensable para los estudiantes de ELE. La falta de conocimiento de colocaciones provoca frases que pueden ser gramaticalmente correctas, pero poco naturales a los nativos como prestar la mano, hacer nacimiento y lluvia pesada ${ }^{2}$. Es decir, el conocimiento de colocaciones mejora la capacidad de expresión de los no nativos. Por eso, especialmente en el ámbito de la enseñanza de inglés, viene insistiéndose en la importancia de enseñar y aprender colocaciones. Por ejemplo, Howarth (1998) ofrece un estudio comparativo de los documentos académicos escritos por nativos de inglés y los textos escritos por no nativos. Comparando los dos tipos de textos, Howarth concluye que «It could therefore be said that native speakers employ about 50 per cent more restricted collocations and idioms (of a particular structural pattern) than learners do, on average» (Howarth, 1998: 177), a lo que añade «moreover, learners need to understand that restricted collocations make up a significant part of a typical native speaker's production in both speech and writing» (Howarth, 1998: 186).

Sin embargo, a pesar de estas sugerencias, las colocaciones del español casi no se enseñan en la realidad. Así es por lo menos en Japón, el lugar donde se ha desarrollado esta investigación. Férnandez Lázaro (2014) explica la situación como sigue:

Pese a que el estudio de las colocaciones es aún relativamente reciente, especialmente en el caso del español, su incidencia en el correcto aprendizaje de una segunda lengua es de sobra reconocida. Sin embargo, la escasez de materiales adaptados a la enseñanza de ELE hace que muchas veces el docente no preste la suficiente atención a las colocaciones ni proporcione a los aprendices la ocasión de reflexionar acerca de este tipo concreto de unidad fraseológica; y, en todo caso, ese acercamiento no se produce nunca antes del nivel B1, siendo lo más habitual relegar el estudio explícito de las colocaciones a los niveles superiores (C1-C2).

Férnandez Lázaro (2014: 2)

Esta escasa atención a la colocación se convirtió en un punto de partida del desarrollo de nuestro material didáctico sobre colocaciones ${ }^{3}$.

\section{CARPA -nuestro material docente-}

Por las razones ya presentadas consideramos que la enseñanza de colocaciones es muy importante. Para enseñarlas de manera eficiente hemos desarrollado un material docente

\footnotetext{
2 Estos errores se sacaron de las composiciones de nuestros estudiantes.

3 El estudio de colocaciones es un entorno lingüístico relativamente nuevo. Por ejemplo, según Koike (2000), el primer estudio que empleó el término colocación en la lingüística española es Írsula Peña (1992).
} 
CARPA (Colocaciones comparando español e inglés). La imagen 1 es muestra del material.
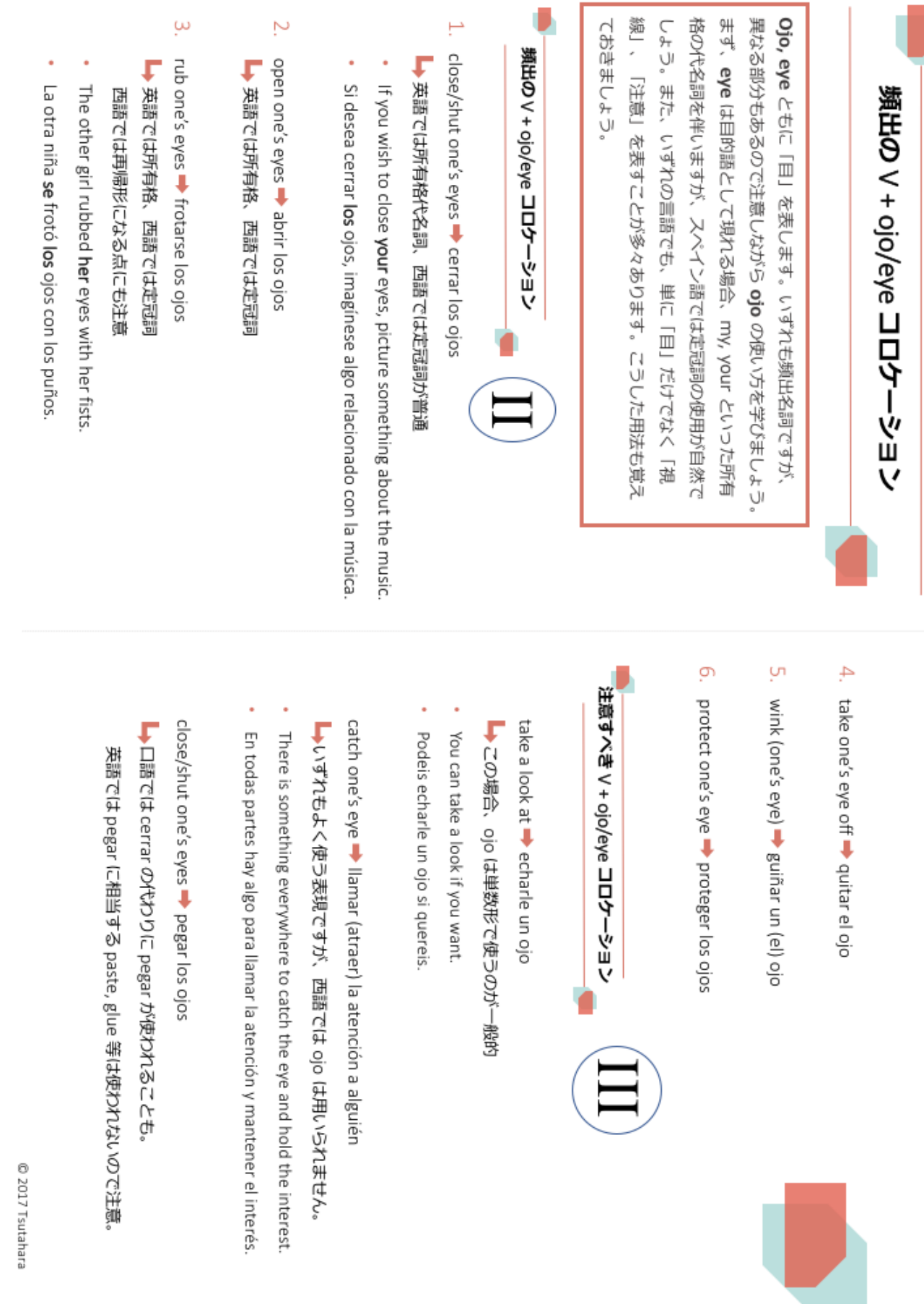

Imagen 1. Muestra de CARPA. Sección de colocaciones de «V+ ojo». 
En esta página de la muestra hemos tratado las colocaciones frecuentes que incluyen el sustantivo ojo, colocaciones de «V + ojo», presentándolas con las colocaciones correspondientes en inglés. Incluyendo el ojo, en el material, tratamos 50 sustantivos y sus colocativos verbales. Además, aparte de los colocativos verbales, presentamos los modificadores típicos de los 50 sustantivos (detallaremos en la sección 2.3.). Cada tipo de colocaciones se presenta en una página y cada página consiste en tres partes: explicaciones generales de la colocación (la parte I en la figura) ${ }^{4}$, las colocaciones más frecuentes (la parte II) y las colocaciones que suelen usarse de manera equivocada (la parte III).

En las secciones siguientes, explicaremos su característica, propiedad, novedad y a qué y a quién se dedica presentando nuestra idea acerca de la enseñanza de colocaciones.

\subsection{Nivel}

Desarrollamos este material básicamente para utilizarlo en las clases de nivel inicial, A1 y A2. Como las colocaciones constituyen gran parte de las comunicaciones de nativos, consideramos beneficioso estar atentos a los usos de colocaciones desde la etapa inicial del aprendizaje. Sin embargo, algunos datos, explicaciones e indicaciones también son útiles para los estudiantes de nivel más alto porque en Japón, y quizás en muchos otros países también, no se hace mucho hincapié en las colocaciones y es muy posible que su conocimiento sea más escaso que el de otras habilidades lingüísticas.

\subsection{Actividad}

Dedicamos unos 15 minutos a las actividades con este material ${ }^{5}$. En los primeros cinco minutos, damos unas explicaciones breves y a continuación los estudiantes hacen frases utilizando las colocaciones que acaban de aprender. Además, en el material hemos puesto varias explicaciones acerca de las colocaciones. Por lo tanto, también puede utilizarse como un libro de texto cuando los estudiantes estudian solos fuera de la clase para ampliar el vocabulario.

\subsection{Colocaciones que se tratan en el material}

Quizás, a la hora de planificar la enseñanza de colocaciones, la cuestión que los docentes

\footnotetext{
${ }^{4}$ El caracter japonés 目 ‘ojo'. denota tanto ojo como eye. En ambos idiomas las respectivas palabras se usan muy frecuentemente, pero sus usos no son siempre idénticos. Por ejemplo, cuando se usa como objeto directo, eye suele venir acompañado de pronombres posesivos como my o your. Al contrario, ojo, se acompaña más frecuentemente de artículos definidos. También hay que tener en cuenta que en ambos casos pueden refierirse tanto a cuidado como a mirada.

${ }^{5}$ En Japón, por lo general, las clases duran noventa minutos.
} 
abordan en primer lugar sea qué colocaciones deben enseñarse. Dado que en un idioma hay muchas palabras y con cada una de ellas se pueden constituir numerosas colocaciones, es provechoso plantearse qué colocaciones son más frecuentes para hacer más útil la enseñanza de las mismas. Esta cuestión también es la que primero abordamos para desarrollar nuestro material docente. Bajo nuestro criterio, decidimos tratar colocaciones que incluyen sustantivos, colocaciones de $« \mathrm{~V}+\mathrm{N}$ (ej. abrir el ojo)» $\mathrm{y} « \mathrm{~N}+$ modificadores (ej. ojo ajeno)». En otros términos, lo que intentamos es ampliar el vocabulario de los estudiantes y mejorar su capacidad de expresión partiendo de sustantivos. Cada sustantivo tiene verbos y modificadores que coaparecen frecuentemente. Por lo tanto, aprender colocaciones de los tipos ya presentados es aprender sustantivos, verbos, adjetivos y otros sustantivos eficazmente.

El núcleo de las colocaciones que íbamos a tratar es el sustantivo. Por eso, el primer paso para crear nuestro material fue elegir los sustantivos más frecuentes en el español actual. Para esta selección empleamos el corpus Corpus del español. De este corpus sacamos los 50 sustantivos que se usaron con mayor frecuencia entre los años 2013-2014. En nuestro material tratamos los sustantivos siguientes:

(1) Año, parte, vida, hombre, mundo, ojo, mano, pelo, cabeza, cuerpo, pie, boca, persona, tipo, lugar, sistema, verdad, proceso, tema, nombre, problema, ciudad, amor, desarrollo, ley, grupo, familia, amigo, uso, equipo, sociedad, punto, situación, nivel, cosa, libro, dinero, empresa, proyecto, dato, comentario, idea, salud, relación, palabra, hijo, muerte, sentido, seguridad, comida

A continuación, para reunir los verbos y modificadores que coaparecen con los 50 sustantivos ya reunidos utilizamos el diccionario combinatorio REDES (Bosque Muñoz 2005) y los corpus European Spanish Web Corpus (Sketch Engine 2011-) y Corpus del español (Davies 2002-). Estos recursos ofrecen los colocativos de las palabras seleccionadas. Consultando cada uno de los 50 sustantivos se sacan verbos y modificadores que forman colocaciones con los sustantivos. Así especificamos las colocaciones más útiles para los estudiantes. Además, la especificación de las colocaciones fue realizada en el corpus ${ }^{6}$, por lo tanto, nuestras colocaciones son las que se utilizan más frecuentemente en el español actual y podrán considerarse como frases vivas y naturales. Resumiendo, CARPA es un material docente con el que se aprenden las colocaciones « $\mathrm{V}+\mathrm{N} » \mathrm{y} « \mathrm{~N}+$ modificadores» que incluyen los 50 sustantivos más frecuentes en el español actual.

\footnotetext{
${ }^{6}$ Ambos corpus ofrecen funciones recopiladora de colocaciones. En el caso de European Spanish Web Corpus se localiza bajo la denominación «sketch difference» y en el de Corpus del español se denomina «Collocates».
} 


\subsection{Colocación y transferencias}

Después de especificar qué colocaciones enseñar a los estudiantes, consideramos cómo enseñarlas y presentarlas en el material. Al final, decidimos presentarlas junto a las colocaciones correspondientes en inglés para que los estudiantes puedan compararlas y facilitar el aprendizaje. Consideramos que esta forma de presentación es la mayor novedad y particularidad del material. En esta sección explicaremos cómo funciona la comparación y por qué hicimos nuestro material así. En síntesis, se trata de aprovechar la transferencia positiva del inglés para facilitar el aprendizaje y también para eliminar la interferencia negativa del mismo idioma, previniendo errores colocacionales.

Es decir, esta forma de nuestro material docente tiene mucho que ver con la transferencia. Transferencia, en este contexto, se entiende como una influencia de otros idiomas que uno está aprendiendo. La transferencia puede afectar al aprendizaje tanto positiva como negativamente. En cuanto a la transferencia positiva, por ejemplo, cuando un estudiante intenta aprender colocaciones, el conocimiento de otros idiomas puede ayudar a los estudiantes si en ese idioma hay colocaciones parecidas. Por ejemplo, para los estudiantes anglófonos, entender y aprender la colocación consultar un libro no es difícil puesto que en su lengua materna hay una colocación léxicamente paralela: to consult a book ${ }^{7}$. En realidad, entre español e inglés hay muchas similitudes. Especialmente, muchos textos de gramática del español publicados en los países anglófonos emplean la estrategia de explicar la gramática comparándola con el correspondiente en inglés para facilitar el aprendizaje $^{8}$. Consideramos que en cuanto a las colocaciones también hay muchas afinidades entre los dos idiomas (como explicaremos más detalladamente en la sección 3) y estamos seguros de que las colocaciones del español pueden enseñarse razonablemente comparándose con las colocaciones en inglés. Explicándose más o menos como "en inglés, como sabéis bien, el verbo to consult se combina con un nombre book. Lo mismo ocurre en el español. El verbo consultar se combina con libro y el significado de la frase es el mismo".

El otro objetivo de hacer comparaciones es prevenir la transferencia negativa del inglés. Entre el español el inglés, aunque hay muchas similitudes, también hay diferencias. A veces, por confiar demasiado en la analogía, los estudiantes cometen errores. Por ejemplo, las frases poco naturales como lluvia pesada (en el sentido de lluvia torrencial) y prestar la mano a alguien son traducciones literales de inglés (heavy rain y to lend one's hand,

\footnotetext{
${ }^{7}$ Esta colocación puede ser difícil para los estudiantes cuyas lenguas maternas son distintas. Por ejemplo, en japonés la traducción literal de consultar un libro, hon ni soudan suru suena sumamente forzado.

${ }^{8}$ cf. Madrigal (1995) y Bregstein (2005).
} 
respectivamente). Para aprovechar la transferencia positiva del inglés y prevenir errores consideramos indispensable que tanto los docentes como los estudiantes tengan bien en cuenta las diferencias colocacionales entre ambos idiomas. Como se ha explicado ya, los japoneses y los estudiantes de muchos otros países pueden sufrir la transferencia negativa de inglés por haber estudiado inglés antes de estudiar español. Por este motivo, hemos establecido una sección de "colocaciones con las que se debe tener cuidado" en nuestro material (la parte III en la imagen 1) y aquí se explican las colocaciones que suelen ser usadas erróneamente.

\section{Datos que avalan la eficacia del material}

La mayor particulalidad de nuestro material es que se emplea la estrategia de comparar colocaciones en español con sus correspondientes en inglés. En esta sección presentamos datos que indica la eficacia de esta estrategia.

En nuestro material, tratamos 50 sustantivos y colocaciones formadas por ellos. El número total de las colocaciones sacadas de REDES y European Spanish Web Corpus suma un total de 2420. Lo que nos llama mucho la atención es que la mayoría de las colocaciónes $(2142 / 2420,88.5 \%)$ es léxicamente paralela a las colocaciones correspondientes en inglés, como consultar libros/to consult books, formar parte/to form part, salvar vida/to save life, poner nombre/to put name, etc. Como la mayoría de las colocaciones en español que tratamos en el material es léxicamente parecida a las correspondientes en inglés, se podría decir que nuestra estrategia de explicar y presentar colocaciones tiene cierta eficacia.

Por otro lado, las 278 colocaciones que son léxicamente distintas del inglés tienen que tratarse con el mayor cuidado tanto en el material como en las clases. Por ejemplo, en los siguientes pares se observa la "discrepancia colocacional".

(2) Ojo morado/black eye (??purple eye, violet eye), echar una mano/to lend a hand (??to pour a hand), pegar ojo/to close eyes (??to paste, glue eye), mano de obra/labour (??hand of work), cuerpo docente/teaching stuff (??teaching body), dar pie a/cause (??to give a foot to), callar la boca/to shut up (??to silence the mouth), persona mayor/elderly person (??major person), etc.

En el español, un hematoma alrededor del ojo se dice ojo morado. En cambio, en inglés no se dice purple eye ni violet eye sino que se dice black eye. Así en los casos presentados, para referirse a los mismos objetos o conceptos en cada idioma se emplean palabras léxicamente distintas. Esto es lo que llamamos discrepancia y estas colocaciones deberán enfatizarse más en las clases. 
También se nota que en muchas de las colocaciones en (2), los sustantivos se usan en sentido figurado. Por ejemplo, en el caso de echar una mano, la mano no se refiere a una parte del cuerpo sino a la ayuda y este tipo de frases suele ser no traducible a otro idioma (pour hand, lie down hand). Estos ejemplos indican que las colocaciones en sentido figurado tienen que enseñarse y aprenderse con el mayor cuidado.

Tambén es provechoso tener en cuenta que a veces una palabra en español se corresponde con dos o más palabras en inglés (y viceversa) y las colocaciones que incluyen estas palabras suelen usarse erróneamente. Por ejemplo, la palabra hair en inglés corresponde con dos palabras en español: pelo y vello. La colocación en inglés black hair, corresponde a pelo negro, sin embargo, en el caso de facial hair, suena raro decir ??pelo facial. Es mucho más natural decir vello facial. Resumiendo, consideramos que las palabras en español que se corresponden con más de dos palabras en inglés o en su lengua materna tienen que tratarse cuidadosamente aclarando sus relaciones, lo que profundizaría el conocimiento de léxico en ambos idiomas.

\section{Conclusión}

En este trabajo hemos defendido la importancia del conocimiento de la colocación para los estudiantes de ELE y hemos propuesto una metodología de enseñanza de la colocación. Según nuestra opinión, el conocimiento del inglés puede facilitar el aprendizaje de colocaciones y las colocaciones del español pueden aprenderse eficazmente comparándose con las correspondientes en inglés. También hemos presentado nuestro material basado en esta idea e introducido la particularidad del material. En la sección 3, hemos mostrado los datos que indican la eficacia de la estrategia.

Una de las tareas que quedan pendientes de un desarrollo futuro es ampliar el número de sustantivos trabajados, así como redactar un material dirigido a estudiantes de nivel más alto. Especialmente, para la redacción del material de nivel más alto, parece que quedan no pocas cuestiones que abordar. Por ejemplo, habrá que establecer un criterio para obtener eficazmente las colocaciones que resulten utiles para los estudiantes de nivel más alto y es necesario considerar cómo presentarlas.

\section{Bibliografía}

Bosque Muñoz, I. (dir). (2005). Redes: Diccionario combinatorio de español contemporáneo. Madrid: Ediciones SM. (REDES).

Bregstein, B. (2005). Easy Spanish Step by Step. New York: McGraw-Hill Education.

DAVIES, M. (2002-). Corpus del español. Recuperado el 27/03/2017 de 
http://www.corpusdelespanol.org/web-dial/.

Corpas Pastor, G. (1997). Manual de fraseología española. Madrid: Gredos.

FERNÁNDEZ LÁZARO, G. (2014). «Enseñanza-aprendizaje de las colocaciones en nivel inicial (A1-A2) ». MarcoELE: Revista de didáctica de español como lengua extranjera, $19,1-16$.

HowArTh, P. (1998). «The phraseology of learner's academic writing». In Cowie, A.P. (ed.). Phraseology (pp. 161-186). Oxford: Oxford University Press.

Írsula PeÑA, J. (1992). «Colocaciones sustantivo-verbo». En Wotjak, G. (ed.). Estudios de lexicografia y metalexicografía del español actual (pp. 159-167). Tubinga: Max Niemeyer Verlag.

KoIKe, K. (2001). Colocaciones léxicas en el español actual: análisis formal y léxicosemántico. Tesis doctoral. Madrid: Universidad de Alcalá y Universidad de Takushoku. Madrigal, M. (1995). Madrigal Magic Key to Spanish. New York: Three Rivers Press. SinclaIR, J. (1991). Corpus, concordance, collocation. Oxford: Oxford University Press. Sketch Engine. (2011-). European Spanish Web Corpus. Recuperado el 27/03/2017 de https://the.sketchengine.co.uk/bonito/corpus/first_form?corpname=preloaded/eseutenten 11_freeling_v4;

StubBs, M. (2001). Words and phrases. Corpus studies of lexical semantics. Oxford: Blackwell. 